REPRESENTATION THEORY

An Electronic Journal of the American Mathematical Society

Volume 3, Pages 127-138 (July 7, 1999)

S $1088-4165(99) 00068-0$

\title{
TRANSFER FACTORS FOR LIE ALGEBRAS
}

\author{
ROBERT E. KOTTWITZ
}

\begin{abstract}
Let $G$ be a quasi-split connected reductive group over a local field of characteristic 0 , and fix a regular nilpotent element in the Lie algebra $\mathfrak{g}$ of $G$. A theorem of Kostant then provides a canonical conjugacy class within each stable conjugacy class of regular semisimple elements in $\mathfrak{g}$. Normalized transfer factors take the value 1 on these canonical conjugacy classes.
\end{abstract}

\section{INTRODUCTION}

Let $k_{0}$ be a local field of characteristic 0 , let $k$ be an algebraic closure of $k_{0}$, and let $\Gamma$ denote the Galois group of $k$ over $k_{0}$. Let $G$ be a connected reductive group over $k_{0}$, with Lie algebra $\mathfrak{g}$. We write $\mathfrak{g}\left(k_{0}\right)$ for the set of $k_{0}$-rational points on $\mathfrak{g}$. Let $(H, \mathcal{H}, s, \xi)$ be endoscopic data [LS87] for $G$. In [LS87] Langlands and Shelstad define transfer factors for $G$ relative to $(H, \mathcal{H}, s, \xi)$, and they conjecture that their transfer factors can be used to define a notion of endoscopic induction (analogous to parabolic induction), associating to any stably invariant distribution on $H\left(k_{0}\right)$ an invariant distribution on $G\left(k_{0}\right)$.

Waldspurger [Wal97] has shown that the conjecture of Langlands and Shelstad would follow from another conjecture, known as the fundamental lemma. Waldspurger's method is to study endoscopy on the Lie algebra $\mathfrak{g}$, and he starts by defining transfer factors for Lie algebras, analogous to those of Langlands and Shelstad.

The main result of this paper gives a new way to express these transfer factors for Lie algebras in the case that the group $G$ is quasi-split over $k_{0}$, which we now assume. In order to state this result precisely we must specify how we are normalizing our transfer factors. Therefore we need to fix a $k_{0}$-splitting $\mathbf{s p l}=$ $\left(B_{0}, T,\left\{X_{\alpha}\right\}\right)$ for $G$. Thus $B_{0}$ is a Borel subgroup of $G$ over $k_{0}, T$ is a maximal $k_{0}$-torus in $B_{0}$, and $\left\{X_{\alpha}\right\}$ is a collection of simple root vectors $X_{\alpha} \in \mathfrak{g}_{\alpha}$, one for each simple root $\alpha$ of $T$ in the Lie algebra of $B_{0}$, having the property that $X_{\sigma \alpha}=\sigma\left(X_{\alpha}\right)$ for all $\sigma \in \Gamma$. (As usual, for any root $\beta$ of $T$ in $\mathfrak{g}$ we write $\mathfrak{g}_{\beta}$ for the corresponding root subspace of $\mathfrak{g}$.)

Waldspurger's factors are analogous to the transfer factors $\Delta\left(\gamma_{H}, \gamma_{G} ; \bar{\gamma}_{H}, \bar{\gamma}_{G}\right)$ on p. 248 of [LS87] with the factor $\Delta_{\text {IV }}$ removed. On the quasi-split group $G$ Langlands and Shelstad also define transfer factors $\Delta_{0}\left(\gamma_{H}, \gamma_{G}\right)$ (again on p. 248 of [LS87]). These depend on the chosen $k_{0}$-splitting spl. The transfer factors $\Delta_{0}^{\prime}\left(X_{H}, X_{G}\right)$ in this paper are complex roots of unity, analogous to $\Delta_{0}\left(\gamma_{H}, \gamma_{G}\right)$ with the factor $\Delta_{\mathrm{IV}}$ removed, and they too depend on the choice of $k_{0}$-splitting.

Received by the editors April 29, 1999.

1991 Mathematics Subject Classification. Primary 22E50; Secondary 11S37.

(c)1999 American Mathematical Society 
We write $\mathfrak{b}_{0}$ for the Lie algebra of the Borel subgroup $B_{0}$. For each simple root $\alpha$ we define a root vector $X_{-\alpha} \in \mathfrak{g}_{-\alpha}$ by requiring that $\left[X_{\alpha}, X_{-\alpha}\right]$ be the coroot for $\alpha$, viewed as an element in the Lie algebra of $T$. We put $X_{-}:=\sum_{\alpha} X_{-\alpha}$, where $\alpha$ runs over the set of simple roots of $T$ in $B_{0}$. Of course $X_{-}$lies in $\mathfrak{g}\left(k_{0}\right)$ and depends on the choice of $k_{0}$-splitting.

The main result of this paper, Theorem 5.1, is that $\Delta_{0}^{\prime}\left(X_{H}, X_{G}\right)=1$ whenever $X_{G}$ lies in $\mathfrak{b}_{0}\left(k_{0}\right)+X_{-}$. By results of Kostant [Kos63] every stable conjugacy class of regular semisimple elements in $\mathfrak{g}\left(k_{0}\right)$ meets the set $\mathfrak{b}_{0}\left(k_{0}\right)+X_{-}$. Since the values of $\Delta_{0}^{\prime}\left(X_{H}, X_{G}\right)$ and $\Delta_{0}^{\prime}\left(X_{H}, X_{G}^{\prime}\right)$ are related by a simple Galois-cohomological factor whenever $X_{G}$ and $X_{G}^{\prime}$ are stably conjugate, this main result also yields a simple formula (see Corollary 5.2) for $\Delta_{0}^{\prime}\left(X_{H}, X_{G}\right)$ for arbitrary $X_{G}$.

The methods used in this paper are variants of ones used in [Lan83], [LS87], [She89]. In particular we rely on a key result of Langlands [Lan83], namely Proposition 5.2. However, since we need this result in a slightly different form, we have included its proof, which is essentially just a rearrangement of the one given by Langlands. The new ingredient in this paper is the connection with Kostant's section. Kostant's section [Kos63] is reviewed in 2.4, following Drinfeld's exposition in a lecture at the IAS in February, 1997.

Throughout this paper we follow the convention that Lie algebras of groups $G$, $B, T$ are denoted by the corresponding gothic letters $\mathfrak{g}, \mathfrak{b}, \mathfrak{t}$, and so on. We also use $N_{G}(T)$ to denote the normalizer in $G$ of $T$. In the first several sections we work over an algebraically closed field $k$ of characteristic 0 ; later we work over a local field $k_{0}$ with algebraic closure $k$.

\section{Review of Kostant's sections of $\mathfrak{g} \rightarrow \mathfrak{t} / W$}

2.1. Basic definitions. Let $G$ be a connected reductive group over an algebraically closed field $k$ of characteristic 0 . We fix a maximal torus $T$ in $G$ and a Borel subgroup $B_{0}$ of $G$ containing $T$; thus $B_{0}=T N_{0}$ where $N_{0}$ denotes the unipotent radical of $B_{0}$. We write $B_{\infty}$ for the unique Borel subgroup of $G$ containing $T$ that is opposed to $B_{0}$; thus $B_{\infty}=T N_{\infty}$ where $N_{\infty}$ denotes the unipotent radical of $B_{\infty}$. For any root $\alpha$ of $T$ in $G$ we write $\mathfrak{g}_{\alpha}$ for the root space of $T$ in $\mathfrak{g}$ corresponding to $\alpha$. Then $\mathfrak{n}_{0}=\bigoplus_{\alpha>0} \mathfrak{g}_{\alpha}$ and $\mathfrak{n}_{\infty}=\bigoplus_{\alpha<0} \mathfrak{g}_{\alpha}$. For each simple positive root $\alpha$ we fix a non-zero root vector $X_{\alpha} \in \mathfrak{g}_{\alpha}$. The triple spl $:=\left(B_{0}, T,\left\{X_{\alpha}\right\}\right)$ is called a splitting of $G$.

Let $\alpha$ be a simple positive root. Let $H_{\alpha}$ be the coroot for $\alpha$ regarded as an element in $\mathfrak{t}$, and fix $X_{-\alpha} \in \mathfrak{g}_{-\alpha}$ by the requirement that $\left[X_{\alpha}, X_{-\alpha}\right]=H_{\alpha}$. There is a unique homomorphism $\phi_{\alpha}: S L(2) \rightarrow G$ whose differential sends the elements

$$
h_{0}=\left[\begin{array}{cc}
1 & 0 \\
0 & -1
\end{array}\right], e_{+}=\left[\begin{array}{ll}
0 & 1 \\
0 & 0
\end{array}\right], e_{-}=\left[\begin{array}{ll}
0 & 0 \\
1 & 0
\end{array}\right] \in \mathfrak{s l}(2)
$$

to $H_{\alpha}, X_{\alpha}, X_{-\alpha}$ respectively.

Let $X_{+}:=\sum_{\alpha} X_{\alpha}$, where the index $\alpha$ runs over all simple positive roots. Let $\mu \in X_{*}(T)$ be the sum of the positive coroots for $T$; recall that $\langle\alpha, \mu\rangle=2$ for every simple positive root $\alpha$. We now decompose $\mathfrak{g}$ as $\mathfrak{g}=\bigoplus_{m} \mathfrak{g}(m)$, where $\mathfrak{g}(m)$ is the $(-2 m)$-th weight subspace

$$
\left\{Z \in \mathfrak{g} \mid \operatorname{Ad}(\mu(a)) Z=a^{-2 m} Z \text { for all } a \in \mathbf{G}_{m}\right\}
$$


for the adjoint action Ad $\circ \mu$ of $\mathbf{G}_{m}$ on $\mathfrak{g}$. For any $d \in \mathbf{Z}$ we put $F^{d} \mathfrak{g}=\bigoplus_{m \geq d} \mathfrak{g}(m)$. Thus $F^{0} \mathfrak{g}=\mathfrak{b}_{\infty}$ and $F^{1} \mathfrak{g}=\mathfrak{n}_{\infty}$. Note also that $X_{+} \in \mathfrak{g}(-1)$ and hence that $\operatorname{ad}\left(X_{+}\right)\left(\mathfrak{n}_{\infty}\right) \subset \mathfrak{b}_{\infty}$

2.2. Chevalley's Theorem. We denote by $k[\mathfrak{g}]$ (respectively, $k[\mathfrak{t}]$ ) the $k$-algebra of polynomial functions on $\mathfrak{g}$ (respectively, $\mathfrak{t}$ ). The adjoint action of $G$ on $\mathfrak{g}$ induces an action of $G$ on $k[\mathfrak{g}]$, and the action of the Weyl group $W$ (of $T$ in $G$ ) on $\mathfrak{t}$ induces an action of $W$ on $k[\mathfrak{t}]$. A theorem of Chevalley (see [CG97] for example) states that the restriction mapping $k[\mathfrak{g}] \rightarrow k[\mathfrak{t}]$ induces an isomorphism $k[\mathfrak{g}]^{G} \rightarrow k[\mathfrak{t}]^{W}$ from the ring of $G$-invariants in $k[\mathfrak{g}]$ to the ring of $W$-invariants in $k[\mathfrak{t}]$. We denote the affine $k$-variety corresponding to $k[\mathfrak{t}]^{W}$ by $\mathfrak{t} / W$. The morphism $u: \mathfrak{g} \rightarrow \mathfrak{t} / W$ dual to $k[\mathfrak{t}]^{W}=k[\mathfrak{g}]^{G} \hookrightarrow k[\mathfrak{g}]$ sends $Z$ to the $W$-orbit in $\mathfrak{t}$ consisting of elements that are $G$-conjugate to the semisimple part $Z_{s}$ of the Jordan decomposition $Z=Z_{s}+Z_{n}$ of $Z$. (Thus $Z_{s}$ is semisimple, $Z_{n}$ is nilpotent, and $\left[Z_{s}, Z_{n}\right]=0$.)

2.3. Regular elements of $\mathfrak{g}$. An element $Z \in \mathfrak{g}$ is said to be regular if the dimension of its centralizer in $\mathfrak{g}$ is equal to the dimension of $\mathfrak{t}$. Recall [Kos63] that $Z$ is regular if and only if the nilpotent part $Z_{n}$ of the Jordan decomposition of $Z$ is a regular element in the centralizer in $\mathfrak{g}$ of the semisimple part $Z_{s}$ of $Z$. The map $Z \mapsto Z_{s}$ induces (see [Kos63]) a bijection from the set of regular $\operatorname{Ad}(G)$-orbits in $\mathfrak{g}$ to the set of semisimple $\operatorname{Ad}(G)$-orbits in $\mathfrak{g}$, and via the map $u$ both sets of orbits can be identified with $\mathfrak{t} / W$.

2.4. Kostant's section. Kostant proved that every element in the affine subspace $\mathfrak{b}_{\infty}+X_{+}$of $\mathfrak{g}$ is regular (see Lemma 10 in [Kos63]). For any $H \in \mathfrak{t} \subset \mathfrak{b}_{\infty}$, the semisimple part of $H+X_{+}$is conjugate to $H$ (see Lemma 11 in [Kos63]), and hence $\mathfrak{t}+X_{+}$meets every regular $\operatorname{Ad}(G)$-orbit in $\mathfrak{g}$.

Now let $\mathfrak{a}$ be any linear subspace of $\mathfrak{b}_{\infty}$ that is complementary to $\operatorname{ad}\left(X_{+}\right)\left(\mathfrak{n}_{\infty}\right)$ and stable under the action Ad $\circ \mu$ of $\mathbf{G}_{m}$ (with $\mu$ as in 2.1). Then Kostant proved (see Remark $19^{\prime}$ in [Kos63]) that $\mathfrak{a}+X_{+}$meets every regular $\operatorname{Ad}(G)$-orbit exactly once, and that the composition of the closed embedding $\mathfrak{a}+X_{+} \hookrightarrow \mathfrak{g}$ and the morphism $u: \mathfrak{g} \rightarrow \mathfrak{t} / W$ is an isomorphism $\mathfrak{a}+X_{+} \rightarrow \mathfrak{t} / W$ of algebraic varieties. Moreover Kostant proved (see Proposition 19 in [Kos63]) that for any $Z \in \mathfrak{t}+X_{+}$ there exists a unique element $n(Z) \in N_{\infty}$ such that $\operatorname{Ad}(n(Z))(Z) \in \mathfrak{a}+X_{+}$, and that the map $Z \rightarrow n(Z)$ is a morphism of algebraic varieties from $\mathfrak{t}+X_{+}$to $N_{\infty}$. But in fact the method of proof of Kostant's Proposition 19 shows more, namely that for any $Z \in \mathfrak{b}_{\infty}+X_{+}$there exists a unique element $n(Z) \in N_{\infty}$ such that $\operatorname{Ad}(n(Z))(Z) \in \mathfrak{a}+X_{+}$, and that the map $Z \rightarrow n(Z)$ is a morphism of algebraic varieties from $\mathfrak{b}_{\infty}+X_{+}$to $N_{\infty}$. It follows that the map $(n, Y) \mapsto \operatorname{Ad}(n)(Y)$ is an isomorphism of varieties from $N_{\infty} \times\left(\mathfrak{a}+X_{+}\right)$to $\mathfrak{b}_{\infty}+X_{+}$.

The results discussed above can be summarized as follows. Every element of $\mathfrak{b}_{\infty}+X_{+}$is regular. The group $N_{\infty}$ acts on $\mathfrak{b}_{\infty}+X_{+}$(by the adjoint action), and the morphism $\mathfrak{b}_{\infty}+X_{+} \rightarrow \mathfrak{t} / W$ (obtained by restricting $u$ to $\mathfrak{b}_{\infty}+X_{+}$) is a principal $N_{\infty}$-bundle. Moreover, this principal bundle admits sections and is therefore trivial.

\section{REVIEW OF THE VARIETY $S$ OF STARS}

3.1. Notation. In this section $G$ is again a connected reductive group over an algebraically closed field $k$ of characteristic 0 . We fix a maximal torus $T$ in $G$. We write $X_{*}(T)$ for the lattice of cocharacters of $T$, and we write $X_{*}(T)_{\mathbf{R}}$ for the real vector space obtained from $X_{*}(T)$ by extending scalars from $\mathbf{Z}$ to $\mathbf{R}$. We write $\mathcal{C}$ for 
the set of Weyl chambers in $X_{*}(T)_{\mathbf{R}}$. For any Weyl chamber $C \in \mathcal{C}$ we write $B_{C}$ for the corresponding Borel subgroup of $G$ containing $T$. Thus any root of $T$ appearing in the unipotent radical of $B_{C}$ takes positive values on the chamber $C$. For any pair $C, D$ of adjacent Weyl chambers, we let $P_{C, D}$ be the unique parabolic subgroup of $G$ that contains both $B_{C}$ and $B_{D}$ and whose Levi component has semisimple rank 1. Thus the Lie algebra of $P_{C, D}$ is the direct sum of the Lie algebra of $B_{C}$ and the root space for the unique root of $T$ that is negative for $C$ and positive for $D$.

We denote by $W$ the Weyl group of $T$ in $G$. The group $W$ acts on the left of $X_{*}(T)$ and $\mathcal{C}$.

3.2. Definition of the variety of stars. We now review the definition of the variety $S$ of stars, introduced by Langlands in [Lan83]. Let $\mathcal{B}$ be the flag variety of $G$. Thus elements of $\mathcal{B}$ are Borel subgroups in $G$, and $G$ acts on the left of $\mathcal{B}$ by conjugation. Consider a map $\mathcal{C} \rightarrow \mathcal{B}$, which we think of as a collection $(B(C))_{C \in \mathcal{C}}$ of Borel subgroups $B(C)$ of $G$ indexed by the set of chambers $C \in \mathcal{C}$. We say that $(B(C))_{C \in \mathcal{C}}$ is a star if for every pair $C, D$ of adjacent chambers there exists $g \in G$ such that $g B(C) g^{-1}=B_{C}$ and $g B(D) g^{-1} \subset P_{C, D}$ (equivalently, for every such pair $C, D$ either $B(C)=B(D)$ or there exists $g \in G$ such that conjugation by $g$ carries the pair $(B(C), B(D))$ into $\left.\left(B_{C}, B_{D}\right)\right)$. The set of stars is a Zariski closed subset of the Cartesian product $\mathcal{B} \times \cdots \times \mathcal{B}$, where the factors in the product are indexed by $\mathcal{C}$. Thus the set $S$ of stars is a projective algebraic variety, and the diagonal left action of $G$ on $\mathcal{B} \times \cdots \times \mathcal{B}$ preserves the subset of stars, so that $G$ acts on the left of $S$. There is also an obvious right action of $W$ on $S$ : an element $w \in W$ sends a star $(B(C))_{C \in \mathcal{C}}$ to the star $C \mapsto B(w(C))$. The actions of $G$ and $W$ on $S$ commute.

A star is said to be regular if $B(C) \neq B(D)$ for every pair $C, D$ of adjacent chambers. The set $S^{0}$ of regular stars is a Zariski open subset of $S$, and it is preserved by the actions of $G$ and $W$. There is an obvious base-point $s_{0} \in S^{0}$, namely the regular star $C \mapsto B_{C}$. The action of $G$ on $S^{0}$ is transitive, and the stabilizer in $G$ of the base-point is $T$, so that $S^{0} \simeq G / T$ as $(G, W)$-varieties.

3.3. Some rational functions $z(C, \beta)$ on $S$. At this point we need to fix a splitting $\left(B_{0}, T,\left\{X_{\alpha}\right\}\right)$ (see 2.1) whose torus component is the torus $T$ we fixed at the beginning of this section. As in 2.1 we write $B_{0}=T N_{0}$ and $B_{\infty}=T N_{\infty}$, where $B_{\infty}$ is the unique Borel subgroup containing $T$ and opposite to $B_{0}$. The group $B_{\infty}$ has a unique open orbit on $\mathcal{B}$, namely the big cell consisting of all Borel subgroups $B \in \mathcal{B}$ opposed to $B_{\infty}$. The Borel subgroup $B_{0}$ lies in the big cell, and the group $N_{\infty}$ acts simply transitively on the big cell, so that every Borel subgroup $B$ opposed to $B_{\infty}$ can be written as $B=n B_{0} n^{-1}$ for a unique element $n \in N_{\infty}$.

Following Langlands [Lan83], we let $S\left(B_{\infty}\right)$ denote the Zariski open subset of $S$ consisting of all stars $C \mapsto B(C)$ such that $B(C)$ is opposed to $B_{\infty}$ for all $C \in \mathcal{C}$. For each pair $(C, \beta)$ consisting of a chamber $C \in \mathcal{C}$ and a root $\beta$ of $T$ that is simple relative to $C$ (that is, $\beta$ is positive on $C$ and its kernel is a wall of $C$ ), Langlands [Lan83] defines a regular function $z(C, \beta)$ on $S\left(B_{\infty}\right)$. This regular function $z(C, \beta)$ depends on the choice of splitting $\left(B_{0}, T,\left\{X_{\alpha}\right\}\right)$. Let $w$ be the unique element in $W$ such that $C=w\left(C_{0}\right)$, where $C_{0}$ is the unique chamber in $\mathcal{C}$ such that $B_{C_{0}}=B_{0}$, and put $\alpha:=w^{-1}(\beta)$; note that $\alpha$ is simple relative to $B_{0}$. The value of the regular function $z(C, \beta)$ at a star $(B(C))$ in $S\left(B_{\infty}\right)$ is defined to be the unique element $z$ in the field $k$ such that

$$
n B(D) n^{-1}=\exp \left(-z X_{-\alpha}\right) B_{0} \exp \left(z X_{-\alpha}\right),
$$


where $D \in \mathcal{C}$ is the unique chamber adjacent to $C$ across the wall defined by $\beta$, and where $n \in N_{\infty}$ is uniquely determined by the condition $n B(C) n^{-1}=B_{0}$.

It is obvious that the right $W$-action on $S$ preserves the subset $S\left(B_{\infty}\right)$. Moreover, the $W$-action permutes the regular functions $z(C, \beta)$. More precisely, for $w \in W$ we have

$$
z(C, \beta) \circ r_{w}=z(w(C), w(\beta)),
$$

where $r_{w}: S\left(B_{\infty}\right) \rightarrow S\left(B_{\infty}\right)$ denotes the right action of $w$.

The left action of $B_{\infty}=T N_{\infty}$ on $S$ preserves the subset $S\left(B_{\infty}\right)$. The regular functions $z(C, \beta)$ on $S\left(B_{\infty}\right)$ are invariant under $N_{\infty}$ and transform as follows under $T$. Let $t \in T$ and let $l_{t}: S\left(B_{\infty}\right) \rightarrow S\left(B_{\infty}\right)$ denote the left action of $t$. We use $(C, \beta)$ to define a $B_{0}$-simple root $\alpha$ as before, so that there exists $w \in W$ carrying $\left(C_{0}, \alpha\right)$ into $(C, \beta)$. Then the transformation law is

$$
z(C, \beta) \circ l_{t}=\alpha(t)^{-1} z(C, \beta) .
$$

We should note that Langlands only defines $z(C, \beta)$ on a certain cross-section for the $N_{\infty}$-action on $S\left(B_{\infty}\right)$, but since there is no essential difference between a regular function on this cross-section and an $N_{\infty}$-invariant regular function on $S\left(B_{\infty}\right)$, we have retained the notation used by Langlands for our extended functions.

3.4. Coordinates on $N_{\infty} \backslash S\left(B_{\infty}\right)$. Let $C \mapsto B(C)$ be a star in $S\left(B_{\infty}\right)$. Thus there exists a unique element $n \in N_{\infty}$ such that $B\left(C_{0}\right)=n B_{0} n^{-1}$. Moreover for each element $w \in W$ there is a unique element $n_{w} \in N_{\infty}$ such that $B\left(w C_{0}\right)=$ $n n_{w} B_{0}\left(n n_{w}\right)^{-1}$. The elements $n_{w}$ can be easily expressed [Lan83] in terms of the values of the regular functions $z(C, \beta)$ at the given star. Indeed, let $w=$ $s_{\alpha_{1}} s_{\alpha_{2}} \ldots s_{\alpha_{p}}$ be a reduced decomposition for $w$, where, as usual, we write $s_{\alpha}$ for the simple reflection associated to the simple root $\alpha$. For $i=1, \ldots, p$ put $C_{i}:=s_{\alpha_{1}} \ldots s_{\alpha_{i}} C_{0}$ and $\beta_{i}=s_{\alpha_{1}} \ldots s_{\alpha_{i-1}} \alpha_{i}$. Thus for $i=1, \ldots, p$ the chambers $C_{i-1}, C_{i}$ are adjacent and separated by $\beta_{i}$. For $i=1, \ldots, p$ let $z_{i}$ denote the value of the function $z\left(C_{i-1}, \beta_{i}\right)$ on the given star. Then it follows immediately from the definition of the regular functions $z\left(C_{i-1}, \beta_{i}\right)$ that

$$
n_{w}=\exp \left(-z_{1} X_{-\alpha_{1}}\right) \exp \left(-z_{2} X_{-\alpha_{2}}\right) \ldots \exp \left(-z_{p} X_{-\alpha_{p}}\right) .
$$

3.5. Refined Bruhat decomposition. We will soon need the following two wellknown statements (part of Bruhat theory). The first statement is that the inclusion of $N_{G}(T)$ in $G$ induces a bijection from $N_{G}(T)$ to $N_{0} \backslash G / N_{0}$. The second statement is that $N_{0} x_{1} N_{0} \cdot N_{0} x_{2} N_{0}=N_{0} x_{1} x_{2} N_{0}$ whenever $x_{1}, x_{2} \in N_{G}(T)$ satisfy the condition that the length of the image of $x_{1} x_{2}$ in $W$ is the sum of the lengths of the images of $x_{1}, x_{2}$.

3.6. Analysis of the $W$-action on $S\left(B_{\infty}\right)$. Now let $C \mapsto B(C)$ be a regular star in $S\left(B_{\infty}\right)$. Denote this star by $s$ and as usual denote the standard star $C \mapsto B_{C}$ by $s_{0}$. We claim that there exists a unique element $g \in N_{\infty} N_{0}$ such that $s=g s_{0}$. Indeed, let $g_{1}$ be any element in $G$ such that $s=g_{1} s_{0}$. Since $B\left(C_{0}\right)$ lies in the big cell, there exists $n \in N_{\infty}$ such that $g_{1} \in n B_{0}$. Modifying the element $g_{1}$ on the right by a suitable element in $T$, we see that there exists $u \in N_{0}$ such that $s=n u s_{0}$. This proves the existence of $g$; its uniqueness is obvious. The uniqueness of the factorization $g=n u$ is also obvious. Note that the element $n$ agrees with the element denoted by $n$ in 3.4 . 
Recall that there is right action of $W$ on $S\left(B_{\infty}\right)$. Let $w \in W$. Then $s^{\prime}:=s w$ is another regular star in $S\left(B_{\infty}\right)$, so there exists unique $g^{\prime}=n^{\prime} u^{\prime} \in N_{\infty} N_{0}$ such that $s^{\prime}=g^{\prime} s_{0}$. Let $x \in G$ be defined by the equation $g^{\prime}=g x$. It is obvious that $x \in N_{G}(T)$ and that $x \mapsto w$ under the canonical surjection $N_{G}(T) \rightarrow W$, and it is also obvious that $x$ depends only on $w$ and the $N_{\infty}$-orbit of $s$. Thus one would expect to be able to express $x$ in terms of $w$ and the values of the regular functions $z(C, \beta)$ on $s$. Such a formula is implicit in [Lan83] (see the proof of Proposition 5.2 of that paper), and is stated explicitly in the next lemma.

As in 3.4 we choose a reduced decomposition $w=s_{\alpha_{1}} \ldots s_{\alpha_{p}}$ and use it and the star $s$ to define scalars $z_{1}, \ldots, z_{p}$. For any simple root $\alpha$ we put

$$
\dot{s}_{\alpha}:=\phi_{\alpha}\left[\begin{array}{cc}
0 & 1 \\
-1 & 0
\end{array}\right]
$$

where $\phi_{\alpha}: S L(2) \rightarrow G$ is the homomorphism defined in 2.1; of course $\dot{s}_{\alpha}$ lies in $N_{G}(T)$ and maps to the simple reflection $s_{\alpha}$ in $W$.

Lemma 3.1. The element $x$ defined above is given by

$$
x=\left(\alpha_{1}^{\vee}\left(z_{1}\right)^{-1} \dot{s}_{\alpha_{1}}\right)\left(\alpha_{2}^{\vee}\left(z_{2}\right)^{-1} \dot{s}_{\alpha_{2}}\right) \ldots\left(\alpha_{p}^{\vee}\left(z_{p}\right)^{-1} \dot{s}_{\alpha_{p}}\right) .
$$

Proof. The star $s^{\prime}$ is equal to $C \mapsto B^{\prime}(C):=B(w(C))$. On the one hand,

$$
B^{\prime}\left(C_{0}\right)=n^{\prime} B_{0}\left(n^{\prime}\right)^{-1} \text {. }
$$

On the other hand,

$$
\begin{aligned}
B^{\prime}\left(C_{0}\right) & =B\left(w\left(C_{0}\right)\right) \\
& =n n_{w} B_{0}\left(n n_{w}\right)^{-1},
\end{aligned}
$$

with $n_{w} \in N_{\infty}$ as in 3.4 (relative to the star $s$ ). Therefore $n^{-1} n^{\prime}=n_{w}$, and hence

$$
x=g^{-1} g^{\prime}=u^{-1} n^{-1} n^{\prime} u^{\prime} \in N_{0} n_{w} N_{0} .
$$

A simple calculation in $S L(2)$ shows that for any simple root $\alpha$

$$
N_{0} \exp \left(-z X_{-\alpha}\right) N_{0}=N_{0} \alpha^{\vee}(z)^{-1} \dot{s}_{\alpha} N_{0} .
$$

Combining this with equation (3.4) and the second statement in section 3.5, we find that

$$
N_{0} n_{w} N_{0}=N_{0} y N_{0}
$$

where

$$
y=\left(\alpha_{1}^{\vee}\left(z_{1}\right)^{-1} \dot{s}_{\alpha_{1}}\right)\left(\alpha_{2}^{\vee}\left(z_{2}\right)^{-1} \dot{s}_{\alpha_{2}}\right) \ldots\left(\alpha_{p}^{\vee}\left(z_{p}\right)^{-1} \dot{s}_{\alpha_{p}}\right) .
$$

Comparing (3.6) and (3.7), we see that $N_{0} x N_{0}=N_{0} y N_{0}$, and it then follows from the first statement in section 3.5 that $x=y$, as we needed to show.

3.7. Some 1-cocycles in $N_{G}(T)$. Now suppose that $G, T, B_{0}, B_{\infty}$ are defined over a subfield $k_{0}$ of $k$ such that $k$ is algebraic over $k_{0}$. Thus $k$ is an algebraic closure of $k_{0}$, and we put $\Gamma:=\operatorname{Gal}\left(k / k_{0}\right)$. We write $\mathfrak{g}\left(k_{0}\right)$ for the subset of $\mathfrak{g}$ consisting of $k_{0}$-rational points. Let $Y \in \mathfrak{g}\left(k_{0}\right)$ and suppose that $Y$ is regular semisimple. We choose an element $H \in \mathfrak{t}$ in the $G$-conjugacy class of $Y$. Of course $H$ need not be defined over $k_{0}$.

There exists $g \in G$, unique up to right multiplication by $T$, such that $Y=$ $\operatorname{Ad}(g)(H)$. For $\sigma \in \Gamma$ put $x_{\sigma}:=g^{-1} \sigma(g)$. Then $\sigma \mapsto x_{\sigma}$ is a 1-cocycle of $\Gamma$ in $N_{G}(T)$, and replacing $g$ by $g t$ (for $t \in T$ ) replaces $x_{\sigma}$ by $t^{-1} x_{\sigma} \sigma(t)$. 
The star $s:=g s_{0}$ is well-defined. We now assume that $s$ lies in $S\left(B_{\infty}\right)$. As in 3.6 we normalize $g$ within its coset $g T$ by insisting that $g$ lie in $N_{\infty} N_{0}$. The next lemma gives a formula for the 1-cocycle $x_{\sigma}$ (obtained from this particular $g$ ) in terms of the values of the functions $z(C, \beta)$ on the star $s$. This lemma is a variant of Proposition 5.2 in [Lan83], and our proof (including that of Lemma 3.1) is a rearrangement of Langlands's.

Lemma 3.2. Let $\sigma \in \Gamma$ and let $w_{\sigma}$ be the image of $x_{\sigma}$ in $W$. Choose a reduced decomposition $w_{\sigma}=s_{\alpha_{1}} \ldots s_{\alpha_{p}}$ of $w_{\sigma}$, and as in section 3.4 use it and the star $s$ to define scalars $z_{1}, \ldots, z_{p}$. Then $x_{\sigma}$ is given by

$$
x_{\sigma}=\left(\alpha_{1}^{\vee}\left(z_{1}\right)^{-1} \dot{s}_{\alpha_{1}}\right)\left(\alpha_{2}^{\vee}\left(z_{2}\right)^{-1} \dot{s}_{\alpha_{2}}\right) \ldots\left(\alpha_{p}^{\vee}\left(z_{p}\right)^{-1} \dot{s}_{\alpha_{p}}\right) .
$$

Proof. Since $N_{\infty}, N_{0}$ are defined over $k_{0}$, the element $\sigma(g)$ also lies in $N_{\infty} N_{0}$. The lemma now follows from Lemma 3.1, applied to the elements $g$ and $g^{\prime}=\sigma(g)$.

\section{1-COCYCLES COMING FROM Kostant's SECTION}

4.1. Goal. Our next goal is to calculate the 1-cocycles of section 3.7 for elements $Y$ lying in the image of Kostant's section $v: \mathfrak{t} / W \rightarrow \mathfrak{g}$. By Lemma 3.2 what we must do is calculate certain values of the functions $z(C, \beta)$.

4.2. Values of $z(C, \beta)$ on stars coming from Kostant's section. Let $H \in \mathfrak{t}^{\prime}$, where $\mathfrak{t}^{\prime}$ denotes the set of regular elements in $\mathfrak{t}$. We write $p: \mathfrak{t} \rightarrow \mathfrak{t} / W$ for the map dual to the inclusion $k[\mathfrak{t}]^{W} \hookrightarrow k[\mathfrak{t}]$. Let $Y$ be any element in $\mathfrak{b}_{\infty}+X_{+}$such that $u(Y)=p(H)$ (where $u: \mathfrak{g} \rightarrow \mathfrak{t} / W$ is as in 2.2). Recall from section 2.4 that the $\operatorname{Ad}\left(N_{\infty}\right)$-orbit of $Y$ is uniquely determined by $H$.

Now choose $g \in G$ such that $Y=\operatorname{Ad}(g)(H)$ and define a regular star $s:=g s_{0}$ (as in 3.7). The star $s$ depends only on $Y$, and the $N_{\infty}$-orbit of $s$ depends only on $H$.

Lemma 4.1. The star $s$ lies in $S\left(B_{\infty}\right)$. The value of the regular function $z(C, \beta)$ on $s$ is $\beta(H)$.

Proof. The construction $H \mapsto s$ is a well-defined map $\delta: \mathfrak{t}^{\prime} \rightarrow N_{\infty} \backslash S$. The right action of $W$ on $S$ induces a right action of $W$ on $N_{\infty} \backslash S$, and we define a right action of $W$ on $\mathfrak{t}^{\prime}$ by converting the usual left action into a right action:

$$
H w:=w^{-1}(H) \text {. }
$$

It is then immediate that our map $\delta: \mathfrak{t}^{\prime} \rightarrow N_{\infty} \backslash S$ is $W$-equivariant.

The truth of the two statements of the lemma depends only on the $N_{\infty}$-orbit of $s$. Therefore we are free to take $Y=H+X_{+}$. We write the star $s$ obtained from $Y$ and $H$ as $C \mapsto B(C)$. It is well-known (and easy to prove) that since $H$ is regular, the map $u \mapsto \operatorname{Ad}(u)(H)$ is an isomorphism from $N_{0}$ to $H+\mathfrak{n}_{0}$. Therefore there exists $u \in N_{0}$ such that $Y=\operatorname{Ad}(u)(H)$. Thus $s=u s_{0}$, and it follows that $B\left(C_{0}\right)=B_{0}$. Since the map $\delta: \mathfrak{t}^{\prime} \rightarrow N_{\infty} \backslash S$ is $W$-equivariant, we conclude that $B(C)$ lies in the $N_{\infty}$-orbit of $B_{0}$ for all chambers $C$, which proves the first statement of the lemma.

Again using the $W$-equivariance of $\delta$, and using (3.2) as well, we see that in order to prove the second statement of the lemma, it is enough to prove that for every simple root $\alpha$ the function $z\left(C_{0}, \alpha\right)$ takes the value $\alpha(H)$ on $s$. So fix a simple root $\alpha$ and let $P_{\alpha}=M_{\alpha} N_{\alpha}$ be the associated parabolic subgroup containing $B_{0}$; 
thus $\mathfrak{m}_{\alpha}$ is spanned by $\mathfrak{t}, \mathfrak{g}_{\alpha}$ and $\mathfrak{g}_{-\alpha}$, and $N_{\alpha}$ is the unipotent radical of $P_{\alpha}$. The homomorphism $\phi_{\alpha}: S L(2) \rightarrow G$ factors through $M_{\alpha}$.

Note that for any scalar $z$

$$
\operatorname{Ad}\left(\exp \left(z X_{\alpha}\right)\right)\left(H+X_{+}\right) \in H+X_{\alpha}+\left[z X_{\alpha}, H\right]+\mathfrak{n}_{\alpha} .
$$

Therefore $\operatorname{Ad}\left(\exp \left(\alpha(H)^{-1} X_{\alpha}\right)\right)\left(H+X_{+}\right)$lies in $H+\mathfrak{n}_{\alpha}$. But (again since $H$ is regular) the map

$$
n_{\alpha} \mapsto \operatorname{Ad}\left(n_{\alpha}\right)(H)
$$

is an isomorphism from $N_{\alpha}$ to $H+\mathfrak{n}_{\alpha}$. Therefore there exists $n_{\alpha} \in N_{\alpha}$ such that

$$
H+X_{+}=\operatorname{Ad}\left(\exp \left(-\alpha(H)^{-1} X_{\alpha}\right) \cdot n_{\alpha}\right)(H) .
$$

It follows from this equality that

$$
B\left(s_{\alpha}\left(C_{0}\right)\right)=\exp \left(-\alpha(H)^{-1} X_{\alpha}\right) \cdot \dot{s}_{\alpha} \cdot B_{0} \cdot\left(\exp \left(-\alpha(H)^{-1} X_{\alpha}\right) \cdot \dot{s}_{\alpha}\right)^{-1} .
$$

A simple calculation in $S L(2)$ then shows that

$$
B\left(s_{\alpha}\left(C_{0}\right)\right)=\exp \left(-\alpha(H) X_{-\alpha}\right) \cdot B_{0} \cdot \exp \left(\alpha(H) X_{-\alpha}\right),
$$

and this (together with the fact that $\left.B\left(C_{0}\right)=C_{0}\right)$ shows that the value of $z\left(C_{0}, \alpha\right)$ on $s$ is $\alpha(H)$, as desired.

4.3. 1-cocycles in $N_{G}(T)$ coming from Kostant's section. We return to the situation of 3.7. We assume further that the family $\left\{X_{\alpha}\right\}$ is defined over $k_{0}$, in the sense that $\sigma\left(X_{\alpha}\right)=X_{\sigma(\alpha)}$ for all $\sigma \in \Gamma$. Thus $\left(B_{0}, T,\left\{X_{\alpha}\right\}\right)$ is a $k_{0}$-splitting in the terminology of [LS87]. Moreover the element $X_{+}=\sum_{\alpha} X_{\alpha}$ lies in $\mathfrak{g}\left(k_{0}\right)$.

To define Kostant's section we need to choose a complementary subspace $\mathfrak{a}$ as in 2.4. Of course we may assume that $\mathfrak{a}$ is defined over $k_{0}$. Then $\mathfrak{a}+X_{+}$is defined over $k_{0}$.

Now let $H \in \mathfrak{t}^{\prime}$ and assume that the image $p(H)$ of $H$ in $\mathfrak{t} / W$ is $k_{0}$-rational. Let $Y$ be any $k_{0}$-rational element of $\mathfrak{b}_{\infty}+X_{+}$such that $u(Y)=p(H)$. It is clear from the discussion above that such a $k_{0}$-rational element exists, and it follows from 2.4 that the $\operatorname{Ad}\left(N_{\infty}\left(k_{0}\right)\right)$-orbit of $Y$ is uniquely determined by $H$. The first statement of Lemma 4.1 implies that the star $s$ defined in 3.7 (for $H, Y$ as above) lies in $S\left(B_{\infty}\right)$. Therefore there exists a unique element $g \in N_{\infty} N_{0}$ such that $Y=\operatorname{Ad}(g)(H)$. As in 3.7 we define a 1-cocycle $x_{\sigma}$ of $\Gamma$ in $N_{G}(T)$ by $x_{\sigma}=g^{-1} \sigma(g)$. Note that this 1-cocycle is independent of the choice of $Y$; it depends only on $H$ and our chosen $k_{0}$-splitting $\mathbf{s p l}=\left(B_{0}, T,\left\{X_{\alpha}\right\}\right)$.

Lemma 4.2. Let $\sigma \in \Gamma$ and let $w_{\sigma}$ be the image of $x_{\sigma}$ in $W$. Choose a reduced decomposition $w_{\sigma}=s_{\alpha_{1}} s_{\alpha_{2}} \ldots s_{\alpha_{p}}$ of $w_{\sigma}$ and for $i=1, \ldots, p$ put $\beta_{i}=$ $s_{\alpha_{1}} s_{\alpha_{2}} \ldots s_{\alpha_{i-1}}\left(\alpha_{i}\right)$. Then

$$
x_{\sigma}=\left(\prod_{i=1}^{p} \beta_{i}^{\vee}\left(\beta_{i}(H)\right)\right)^{-1} \cdot \dot{w}_{\sigma},
$$

where $\dot{w}_{\sigma}=\dot{s}_{\alpha_{1}} \ldots \dot{s}_{\alpha_{p}}$.

Proof. This follows immediately from Lemmas 3.2 and 4.1.

However the 1-cocycle we really need is a variant of $x_{\sigma}$. We replace $\mathfrak{b}_{\infty}+X_{+}$ by $\mathfrak{b}_{0}+X_{-}$, where $X_{-}:=\sum_{\alpha} X_{-\alpha}$, the sum ranging over simple roots $\alpha$. In this context Kostant's theory provides an isomorphism (over $k_{0}$ ) from the quotient of $\mathfrak{b}_{0}+X_{-}$by the adjoint action of $N_{0}$ to the space $\mathfrak{t} / W$. 
Let $H \in \mathfrak{t}^{\prime}$ be as above and let $Z$ be any $k_{0}$-rational element of $\mathfrak{b}_{0}+X_{-}$such that $u(Z)=p(H)$. Let $h$ be any element of $G$ such that $Z=\operatorname{Ad}(h)(H)$. Define a 1-cocycle $y_{\sigma}$ of $\Gamma$ in $N_{G}(T)$ by $y_{\sigma}=h^{-1} \sigma(h)$. Replacing $h$ by $h t$, for $t \in T$, has

the effect of replacing $y_{\sigma}$ by $t^{-1} y_{\sigma} \sigma(t)$. Thus the 1-cocycle $y_{\sigma}$ is well-defined up to such equivalences.

Lemma 4.3. For a suitable choice of $h$, the 1-cocycle $y_{\sigma}$ is given by the following formula. Let $\sigma \in \Gamma$ and let $w_{\sigma}$ be the image of $y_{\sigma}$ in $W$. Choose a reduced decomposition $w_{\sigma}=s_{\alpha_{1}} s_{\alpha_{2}} \ldots s_{\alpha_{p}}$ of $w_{\sigma}$ and for $i=1, \ldots, p$ put $\beta_{i}=s_{\alpha_{1}} s_{\alpha_{2}} \ldots s_{\alpha_{i-1}}\left(\alpha_{i}\right)$. Then $y_{\sigma}$ is given by

$$
y_{\sigma}=\left(\prod_{i=1}^{p} \beta_{i}^{\vee}\left(\beta_{i}(H)\right)\right) \cdot \dot{w}_{\sigma}
$$

where $\dot{w}_{\sigma}=\dot{s}_{\alpha_{1}} \ldots \dot{s}_{\alpha_{p}}$.

Proof. Let $\theta$ be the automorphism of $G$ that induces -1 on $\mathfrak{t}$ and exchanges $X_{\alpha}$ and $X_{-\alpha}$ for all simple roots $\alpha$. It is clear that $\theta$ is defined over $k_{0}$ and that its square is the identity. Since $\theta$ exchanges $\mathfrak{b}_{\infty}+X_{+}$and $\mathfrak{b}_{0}+X_{-}$, it is clear that we get a suitable 1-cocycle $y_{\sigma}$ by applying $\theta$ to the 1 -cocycle $x_{\sigma}$ associated to $\theta(H)=-H$. Note that $\theta\left(\dot{s}_{\alpha}\right)=\alpha^{\vee}(-1) \dot{s}_{\alpha}$ for any simple root $\alpha$ (a simple calculation in $S L(2)$ ). The desired result then follows from the previous lemma.

\section{Transfer factors $\Delta_{0}^{\prime}\left(X_{H}, X_{G}\right)$ ON $\mathfrak{g}$}

5.1. Notation. As in 3.7 we assume that $G$ is quasi-split over $k_{0}$, and we fix a $k_{0}$-splitting $\mathbf{s p l}=\left(B_{0}, T,\left\{X_{\alpha}\right\}\right)$ for $G$. We assume further that $k_{0}$ is a local field (of characteristic 0 ). Let $(H, \mathcal{H}, s, \xi)$ be endoscopic data for $G$ (see [LS87]).

5.2. General discussion of transfer factors. Following Waldspurger [Wal97] we consider transfer factors on $\mathfrak{g}$ analogous to the transfer factors on $G$ defined by Langlands and Shelstad [LS87]. Waldspurger's factors are analogous to the transfer factors $\Delta\left(\gamma_{H}, \gamma_{G} ; \bar{\gamma}_{H}, \bar{\gamma}_{G}\right)$ on p. 248 of [LS87] with the factor $\Delta_{\text {IV }}$ removed.

On the quasi-split group $G$ Langlands and Shelstad also define transfer factors $\Delta_{0}\left(\gamma_{H}, \gamma_{G}\right)$ (again on p. 248 of [LS87]). These depend on the chosen $k_{0}$-splitting spl. The transfer factors $\Delta_{0}^{\prime}\left(X_{H}, X_{G}\right)$ in this paper are analogous to $\Delta_{0}\left(\gamma_{H}, \gamma_{G}\right)$ with the factor $\Delta_{\mathrm{IV}}$ removed.

5.3. $a$-data. Let $T_{H}$ be a maximal torus in $H$. There is a canonical $G$-conjugacy class of embeddings $T_{H} \rightarrow G$, and this $G$-conjugacy class contains members that are defined over $k_{0}$. Fix such a $k_{0}$-embedding $T_{H} \rightarrow G$, and let $T_{G}$ denote the image of $T_{H}$ in $G$, a maximal torus of $G$, defined over $k_{0}$. We identify $T_{H}$ with $T_{G}$, so that the set $R_{H}$ of roots of $T_{H}$ in $H$ becomes a subset of the set $R_{G}$ of roots of $T_{G}$ in $G$.

Recall from [LS87], 2.2 that $a$-data for $T_{G}$ consists of elements $a_{\alpha} \in k^{\times}$, one for each $\alpha \in R_{G}$, satisfying

1. $a_{\sigma \alpha}=\sigma\left(a_{\alpha}\right)$ for all $\alpha \in R_{G}$ and all $\sigma \in \Gamma$,

2. $a_{-\alpha}=-a_{\alpha}$ for all $\alpha \in R_{G}$.

We now choose $a$-data for $T_{G}$.

Let $G_{\mathrm{sc}}$ denote the simply connected cover of the derived group of $G$, and let $T_{G}^{\mathrm{sc}}$ denote the inverse image of $T_{G}$ under the canonical homomorphism from $G_{\mathrm{sc}}$ to $G$. Recall that Langlands and Shelstad (see 2.3 in [LS87]) define an invariant 
$\lambda\left(T_{G}^{\mathrm{sc}}\right) \in H^{1}\left(k_{0}, T_{G}^{\mathrm{sc}}\right)$, which depends on spl and the $a$-data $\left\{a_{\alpha}\right\}$, as well as $T_{G}$. We denote by $\lambda\left(T_{G}\right) \in H^{1}\left(k_{0}, T_{G}\right)$ the image of $\lambda\left(T_{G}^{\mathrm{sc}}\right)$ under the map induced by the canonical homomorphism $T_{G}^{\mathrm{sc}} \rightarrow T_{G}$.

5.4. $\chi$-data. For any root $\alpha \in R_{G}$ we let $k_{\alpha}$ (respectively, $k_{ \pm \alpha}$ ) denote the field of definition of $\alpha$ (respectively, the set $\{\alpha,-\alpha\}$ ). Thus $k_{0} \subset k_{ \pm \alpha} \subset k_{\alpha} \subset k$ and $\left[k_{\alpha}: k_{ \pm \alpha}\right]$ is 1 or 2 . As in [LS87], if [ $\left.k_{\alpha}: k_{ \pm \alpha}\right]=2$, we say that $\alpha$ (and its $\Gamma$-orbit in $R_{G}$ ) is symmetric, and we let $\chi_{\alpha}$ denote the quadratic character on $k_{ \pm \alpha}^{\times}$associated to the quadratic extension $k_{\alpha} / k_{ \pm \alpha}$ by local classfield theory. Transfer factors on $\mathfrak{g}$ are simpler than those on $G$, in that it is not necessary to extend $\chi_{\alpha}$ to a quasicharacter on $k_{\alpha}^{\times}$. Consequently, when defining transfer factors on $\mathfrak{g}$, it is irrelevant whether or not $\mathcal{H}$ is isomorphic to ${ }^{L} H$.

5.5. Definition of $\Delta_{0}^{\prime}\left(X_{H}, X_{G}\right)$. Let $X_{H} \in \mathfrak{t}_{H}$ and assume that its image $X_{G}$ in $\mathfrak{t}_{G}$ is regular. We are going to define our transfer factor $\Delta_{0}^{\prime}\left(X_{H}, X_{G}\right)$ by

$$
\Delta_{0}^{\prime}\left(X_{H}, X_{G}\right)=\Delta_{\mathrm{I}}\left(X_{H}, X_{G}\right) \Delta_{\mathrm{II}}\left(X_{H}, X_{G}\right),
$$

where the factors $\Delta_{\mathrm{I}}\left(X_{H}, X_{G}\right), \Delta_{\mathrm{II}}\left(X_{H}, X_{G}\right)$, to be defined below, are analogous to the factors $\Delta_{\mathrm{I}}\left(\gamma_{H}, \gamma_{G}\right), \Delta_{\mathrm{II}}\left(\gamma_{H}, \gamma_{G}\right)$ of [LS87].

5.6. Definition of $\Delta_{\mathrm{I}}\left(X_{H}, X_{G}\right)$. Let $\hat{T}_{G}$ denote the complex torus dual to $T_{G}$ (Langlands duality). The element $s$ appearing in our endoscopic data is a $\Gamma$-fixed element in the center of the Langlands dual group $\hat{H}$ of $H$, and thus can be regarded as a $\Gamma$-fixed element $\mathbf{s}_{T_{G}}$ of $\hat{T}_{H}=\hat{T}_{G}$. There is a Tate-Nakayama pairing (see [Kot86] for example)

$$
\langle\cdot, \cdot\rangle: H^{1}\left(k_{0}, T_{G}\right) \times \hat{T}_{G}^{\Gamma} \rightarrow \mathbf{C}^{\times},
$$

where $\hat{T}_{G}^{\Gamma}$ denotes the group of fixed points of $\Gamma$ in $\hat{T}_{G}$. We define $\Delta_{\mathrm{I}}$ by

$$
\Delta_{\mathrm{I}}\left(X_{H}, X_{G}\right):=\left\langle\lambda\left(T_{G}\right), \mathbf{s}_{T_{G}}\right\rangle .
$$

5.7. Definition of $\Delta_{\mathrm{II}}\left(X_{H}, X_{G}\right)$. We define $\Delta_{\mathrm{II}}$ by

$$
\Delta_{\mathrm{II}}\left(X_{H}, X_{G}\right):=\prod_{\alpha} \chi_{\alpha}\left(\frac{\alpha\left(X_{G}\right)}{a_{\alpha}}\right),
$$

where the product is taken over a set of representatives for the symmetric orbits of $\Gamma$ in the set $R_{G} \backslash R_{H}$.

5.8. Discussion of $\Delta_{0}^{\prime}\left(X_{H}, X_{G}\right)$. It is immediate from (the proof of) Lemma 3.2.C of [LS87] that $\Delta_{0}^{\prime}\left(X_{H}, X_{G}\right)$ is independent of the choice of $a$-data. Thus $\Delta_{0}^{\prime}\left(X_{H}, X_{G}\right)$ depends only on the choice of $k_{0}$-splitting spl.

Put $\Delta_{0}^{\prime}\left(\gamma_{H}, \gamma_{G}\right):=\Delta_{0}\left(\gamma_{H}, \gamma_{G}\right) \cdot \Delta_{\mathrm{IV}}\left(\gamma_{H}, \gamma_{G}\right)^{-1}$, with $\Delta_{0}$ and $\Delta_{\mathrm{IV}}$ as in [LS87]. It is easy to see that for $X_{H}$ sufficiently close to 0

$$
\Delta_{0}^{\prime}\left(X_{H}, X_{G}\right)=\Delta_{0}^{\prime}\left(\exp \left(X_{H}\right), \exp \left(X_{G}\right)\right) .
$$

Moreover it is obvious that

$$
\Delta_{0}^{\prime}\left(a^{2} X_{H}, a^{2} X_{G}\right)=\Delta_{0}^{\prime}\left(X_{H}, X_{G}\right)
$$

for all $a \in k_{0}^{\times}$. These two properties characterize the transfer factors $\Delta_{0}^{\prime}$ on $\mathfrak{g}$. 
Now suppose that $X_{G}^{\prime} \in \mathfrak{g}\left(k_{0}\right)$ is stably conjugate to $X_{G}$, so that there exists $h \in G$ such that $\operatorname{Ad}(h)\left(X_{G}^{\prime}\right)=X_{G}$. Then $\sigma \mapsto h \sigma(h)^{-1}$ is a 1-cocycle of $\Gamma$ in $T_{G}$ whose class we denote by $\operatorname{inv}\left(X_{G}, X_{G}^{\prime}\right)$. Then

$$
\Delta_{0}^{\prime}\left(X_{H}, X_{G}^{\prime}\right)=\Delta_{0}^{\prime}\left(X_{H}, X_{G}\right) \cdot\left\langle\operatorname{inv}\left(X_{G}, X_{G}^{\prime}\right), s_{T_{G}}\right\rangle^{-1} .
$$

This follows from Lemmas 3.2.B and 3.4.A of [LS87], or rather from their (easy) Lie algebra analogs.

5.9. Main result. As above we use our fixed splitting spl to define transfer factors $\Delta_{0}^{\prime}\left(X_{H}, X_{G}\right)$ on $\mathfrak{g}$ and to define an element $X_{-} \in \mathfrak{g}\left(k_{0}\right)$.

Theorem 5.1. The transfer factor $\Delta_{0}^{\prime}\left(X_{H}, X_{G}\right)$ is equal to 1 whenever $X_{G}$ lies in the set of $k_{0}$-rational elements in $\mathfrak{b}_{0}+X_{-}$.

Proof. Note that $a_{\alpha}:=\alpha\left(X_{G}\right)$ is a valid choice of $a$-data for $T_{G}$. With this choice of $a$-data it is obvious that $\Delta_{\mathrm{II}}\left(X_{H}, X_{G}\right)=1$, and what we must show is that $\Delta_{\mathrm{I}}\left(X_{H}, X_{G}\right)=1$. Since this must be true for all endoscopic data, we must show that $\lambda\left(T_{G}^{\mathrm{sc}}\right)=1$ for this particular choice of $a$-data. It is harmless to assume that $G=G_{\mathrm{sc}}$, and we do so in order to simplify notation. Choose $h \in G$ and $H \in \mathfrak{t}$ such that $X_{G}=\operatorname{Ad}(h)(H)$. (Since the endoscopic data are now irrelevant, it should cause no confusion to use our usual convention of denoting elements of $\mathfrak{t}$ by $H$.) Thus $h T h^{-1}=T_{G}$ and we use the inner automorphism $x \mapsto h x h^{-1}$ to identify $T$ with $T_{G}$ over $k$. As in $4.3 y_{\sigma}:=h^{-1} \sigma(h)$ is a 1-cocycle of $\Gamma$ in $N_{G}(T)$, and we denote by $w_{\sigma}$ the image of $y_{\sigma}$ in the Weyl group $W$. On p. 231 of [LS87] Langlands and Shelstad define a 1-cocycle

$$
m_{\sigma}:=\left(\prod_{\beta} \beta^{\vee}\left(a_{\beta}\right)\right) \cdot \dot{w}_{\sigma}
$$

of $\Gamma$ in $N_{G}(T)$, where $\dot{w}_{\sigma}$ is defined as in Lemma 4.3 , and where the product is taken over all positive roots $\beta$ of $T$ in $G$ such that $w_{\sigma}^{-1}(\beta)$ is negative. We are using our identification of $T$ and $T_{G}$ over $k$ to view $\beta$ as a root of $T_{G}$, so that $a_{\beta}$ is defined. For our particular choice of $a$-data, we have $a_{\beta}=\beta(H)$, and therefore $m_{\sigma}$ is exactly the same as the 1-cocycle appearing in Lemma 4.3. It follows from Lemma 4.3 that by choosing $h$ correctly within its coset $h T$, we may assume that

$$
h^{-1} \sigma(h)=m_{\sigma}
$$

Langlands and Shelstad now define a 1-cocycle of $\Gamma$ in $T_{G}$ by $\sigma \mapsto h m_{\sigma} \sigma(h)^{-1}$, and they define $\lambda\left(T_{G}^{\mathrm{sc}}\right)$ to be the class of this 1-cocycle. Since (5.2) shows that this 1-cocycle is trivial, we see that $\lambda\left(T_{G}^{\mathrm{sc}}\right)$ is trivial, as desired.

Corollary 5.2. The transfer factor $\Delta_{0}^{\prime}\left(X_{H}, X_{G}\right)$ is equal to $\left\langle\operatorname{inv}\left(X_{G}, X_{G}^{\prime}\right), s_{T_{G}}\right\rangle$, where $X_{G}^{\prime}$ is any $k_{0}$-rational element in $\mathfrak{b}_{0}+X_{-}$that is stably conjugate to $X_{G}$.

Proof. This follows from the theorem together with (5.1).

\section{REFERENCES}

[CG97] N. Chriss and V. Ginzburg, Representation theory and complex geometry, Birkhäuser, Boston, 1997. MR 98i:22021

[Kos63] B. Kostant, Lie group representations on polynomial rings, Amer. J. Math. 85 (1963), 327-404. MR 28:1252

[Kot86] R. Kottwitz, Stable trace formula: elliptic singular terms, Math. Ann. 275 (1986), 365399. MR 88d:22027 
[Lan83] R. P. Langlands, Orbital integrals on forms of SL(3), I, Amer. J. Math. 105 (1983), 465-506. MR 86d:22012

[LS87] R. Langlands and D. Shelstad, On the definition of transfer factors, Math. Ann. 278 (1987), 219-271. MR 89c:11172

[She89] D. Shelstad, A formula for regular unipotent germs, Astérisque 171-172 (1989), 275-277. MR 91b:22012

[Wal97] J.-L. Waldspurger, Le lemme fondamental implique le transfert, Compositio Math. 105 (1997), 153-236. MR 98h:22023

Department of Mathematics, University of Chicago, 5734 University Avenue, Chicago, ILLINOIS 60637

E-mail address: kottwitz@math.uchicago.edu 